\title{
Ultrafast Cathodic Exfoliation of Few-Layer Black Phosphorus in Aqueous Solution
}

Fei Luo, ${ }^{\dagger}$ Dongya Wang, ${ }^{\dagger}$ Jindong Zhang, ${ }^{\dagger}$ Xiaona $\mathrm{Li},{ }^{\dagger}$ Dongmei Liu $,{ }^{\dagger} \mathrm{Hai} \mathrm{Li},{ }^{\dagger}$ Min Lu, ${ }^{* \dagger}{ }^{\dagger}$ Xiaoji Xie, ${ }^{*}, \dagger$ Ling Huang, ${ }^{\dagger}$ and Wei Huang ${ }^{\dagger, \dagger}$

'Key Laboratory of Flexible Electronics (KLOFE), Institute of Advanced Materials (IAM), Nanjing Tech University (NanjingTech), Nanjing 211816, China

*Shaanxi Institute of Flexible Electronics, Northwestern Polytechnical University, Xi'an 710072, China

Corresponding Author

*E-mail: iammlv@njtech.edu.cn (M.L.), iamxjxie@njtech.edu.cn (X.X.). 

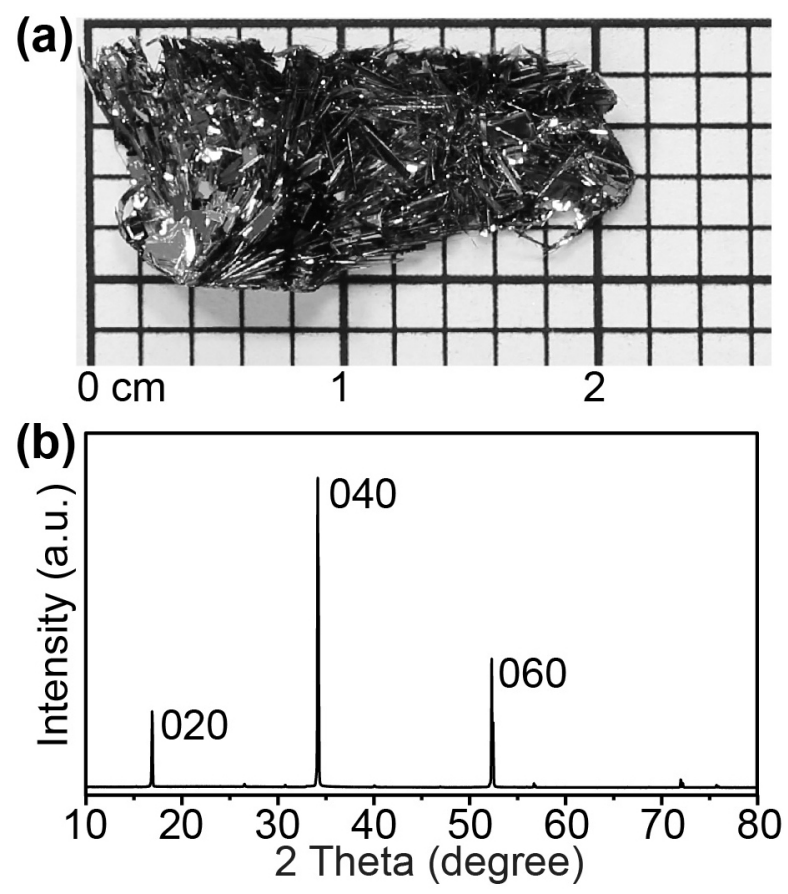

Figure S1. (a) Typical photo of the as-prepared bulk BP crystals. The BP crystals were placed on the centimetre grid paper. (b) Corresponding powder XRD pattern of the obtained BP crystals. 


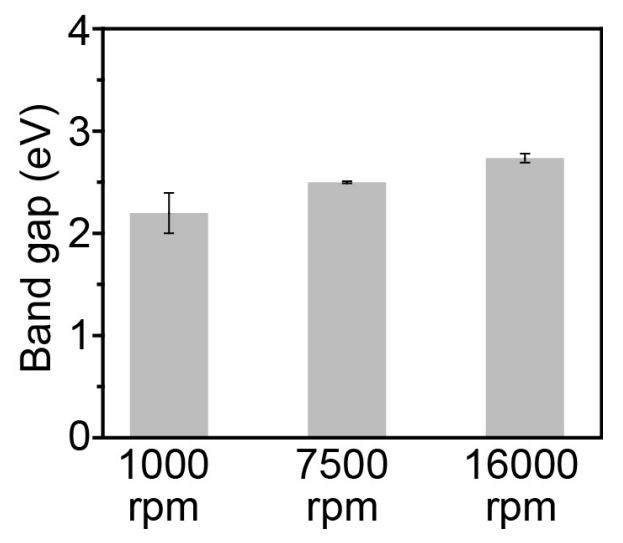

Figure S2. Optical band gaps of the few-layer BP dispersions obtained by gradient centrifugation. Note that the optical band gaps were derived from the UV-vis spectroscopy analysis, and the dispersions of few-layer BP flakes were denoted by the rate of centrifugation. The bulk BP crystals were exfoliated in the presence of an aqueous solution of CTAC $(0.5 \mathrm{M})$, under a voltage of $-30 \mathrm{~V}$, at $50{ }^{\circ} \mathrm{C}$, and the exfoliation lasted for 30 minutes. 

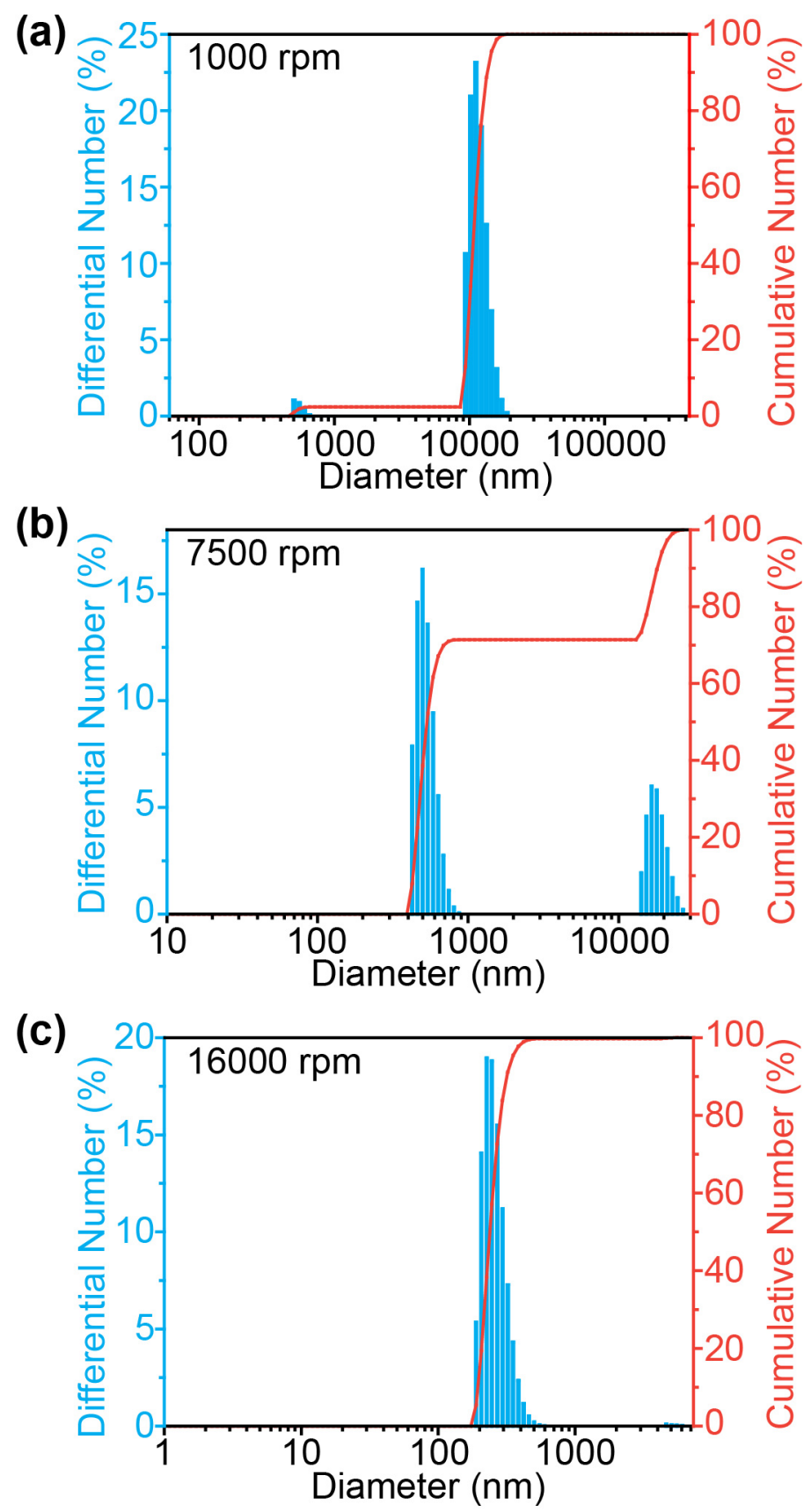

Figure S3. Size distributions of few-layer BP flakes separated by gradient centrifugation. Note that the few-layer BP flakes were denoted by the rate of centrifugation, redispersed in isopropanol, and analyzed by dynamic light scatting. The bulk BP crystals were exfoliated in the presence of an aqueous solution of CTAC $(0.5 \mathrm{M})$, under a voltage of $-30 \mathrm{~V}$, at 50 ${ }^{\circ} \mathrm{C}$, and the exfoliation lasted for 30 minutes. 


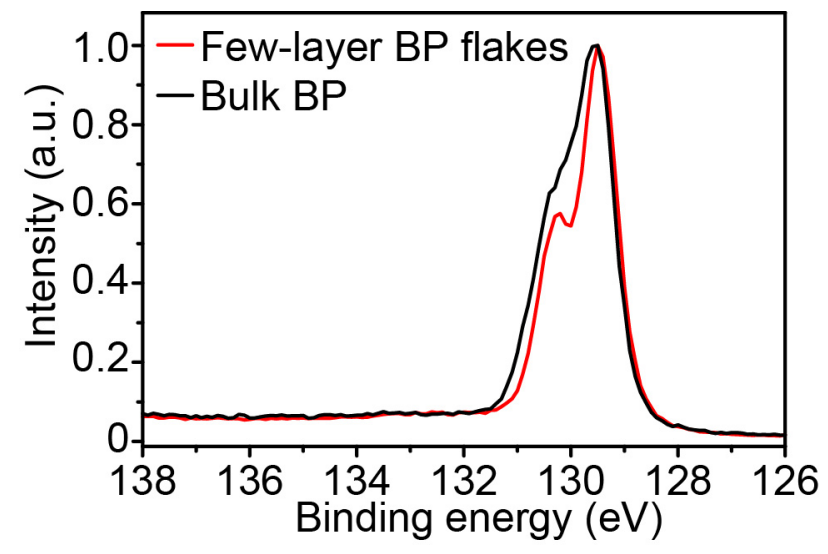

Figure S4. Normalized P 2p XPS spectra of bulk BP crystals and as-exfoliated fewlayer BP flakes. 


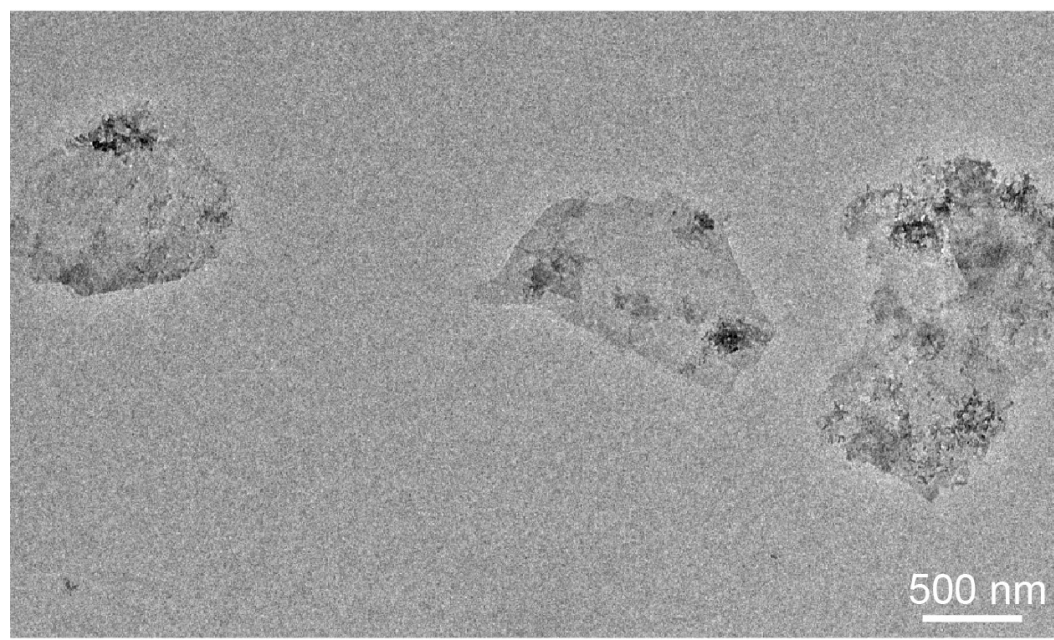

Figure S5. TEM image of the few-layer BP flakes obtained by centrifugation at a rate of $7500 \mathrm{rpm}$. 


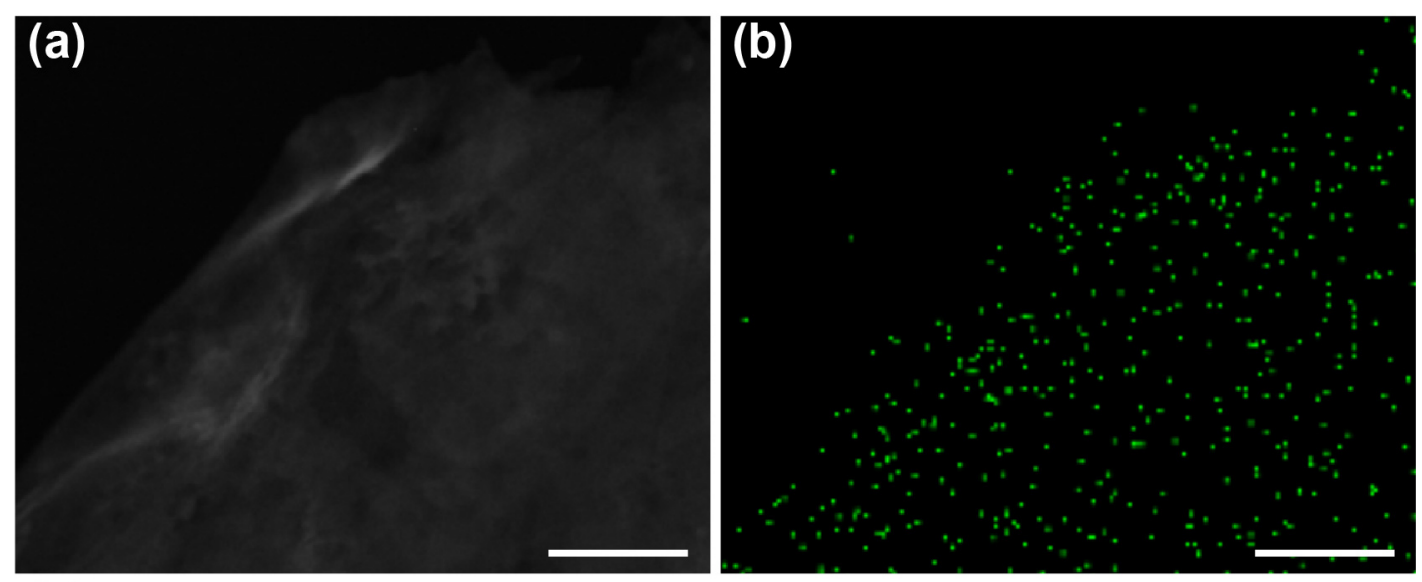

(c)

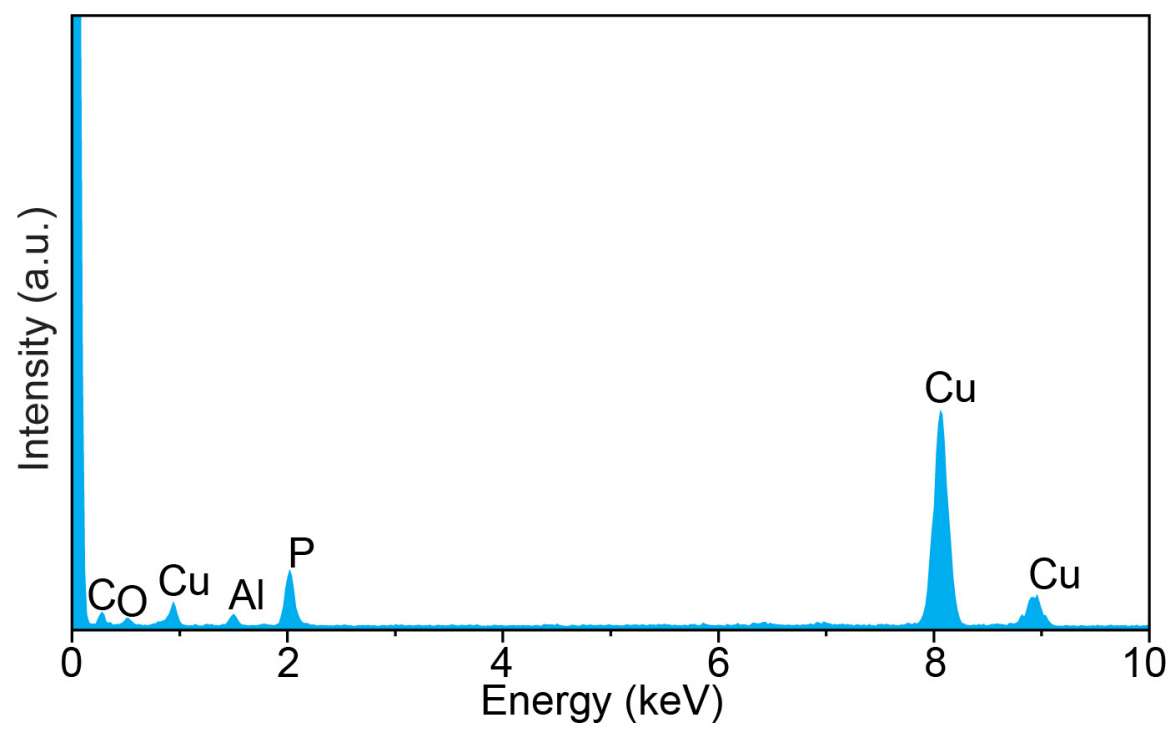

Figure S6. $(a, b)$ Dark-field scanning transmission electron microscopy (STEM) image and corresponding elemental mapping (P element) of a few-layer BP flake obtained by centrifugation at a rate of $7500 \mathrm{rpm}$. Scale bars in $(\mathrm{a}, \mathrm{b})$ are $100 \mathrm{~nm}$. (c) Corresponding EDX spectrum of the few-layer BP flake. It should be mentioned that the appearance of $\mathrm{Cu}$ and $\mathrm{Al}$ in $(\mathrm{C})$ is due to the TEM grid and sample holder. Meanwhile, the appearance of $\mathrm{C}$ and $\mathrm{O}$ elements can be due to the adsorbed molecules (e.g. solvents) and the hydrocarbon contaminations in TEM chamber. 

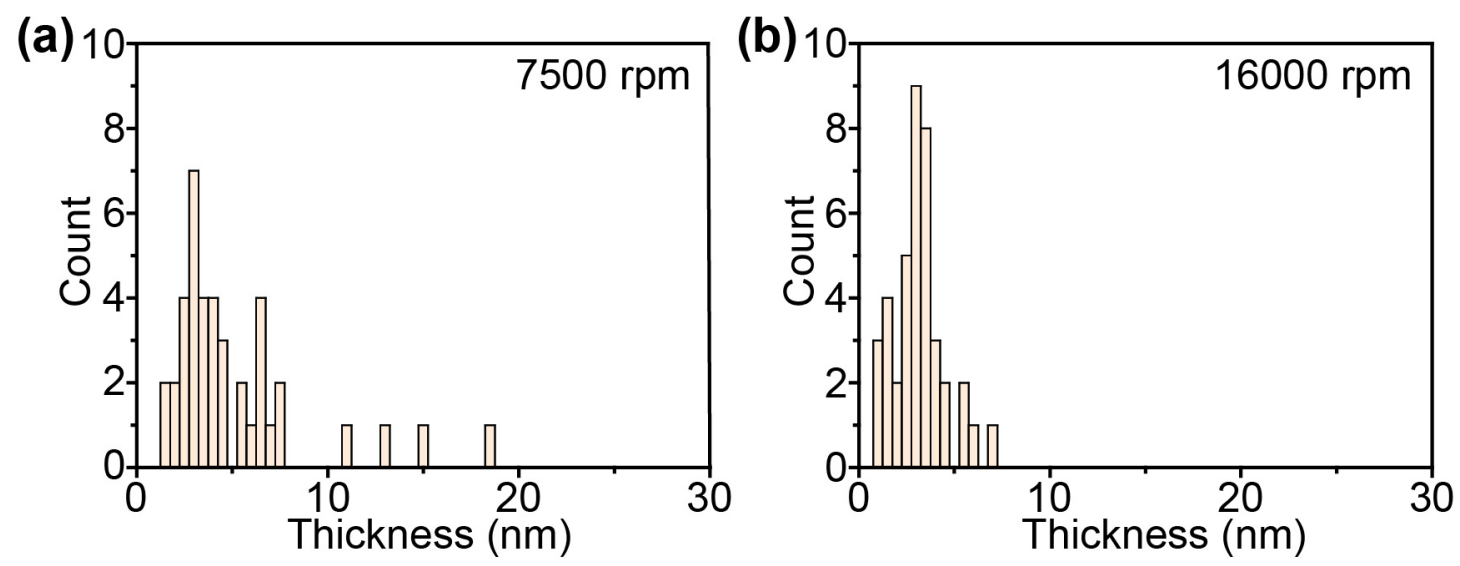

Figure S7. Thickness distribution histograms derived from AFM measurements for the few-layer BP flakes obtained by centrifugation at (a) $7500 \mathrm{rpm}$ and (b) $16000 \mathrm{rpm}$, respectively. 

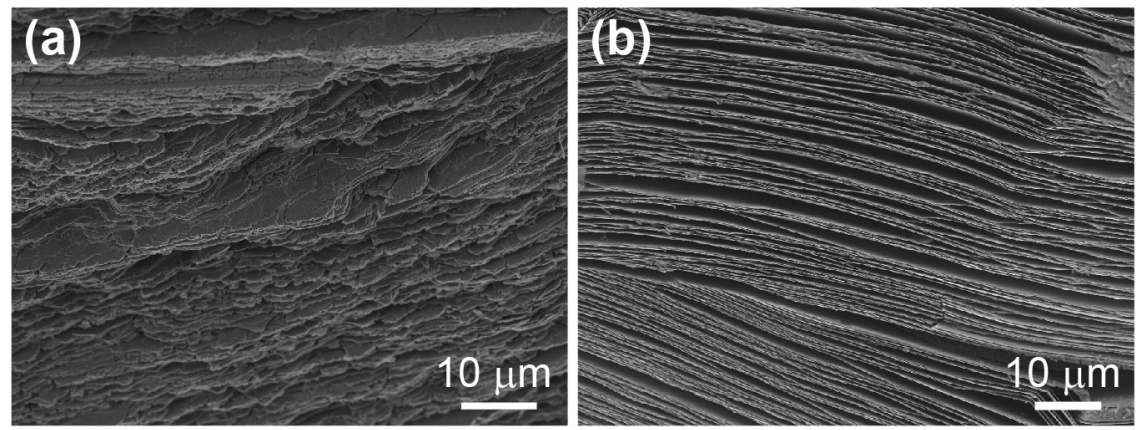

Figure S8. Large scale SEM images of the bulk BP crystals after applying voltages of (a) $-20 \mathrm{~V}$ and (b) $-30 \mathrm{~V}$ for 10 minutes in a CTAC solution $(0.5 \mathrm{M})$ at $50{ }^{\circ} \mathrm{C}$. Note that the bulk BP crystals were thoroughly washed with water and dried under vacuum before the observation in a scanning electron microscope. 

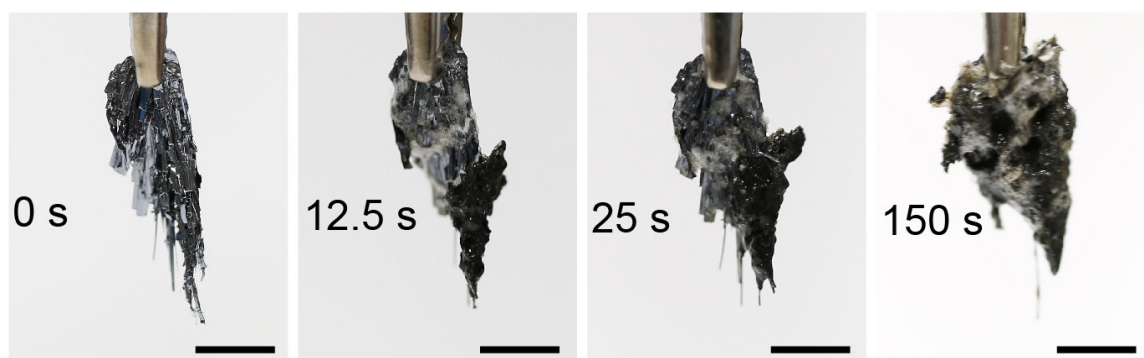

Figure S9. Typical photos of the bulk BP crystals after applying a voltage of -30 V for different time in a CTAC solution $(0.5 \mathrm{M})$ at $50{ }^{\circ} \mathrm{C}$. Scale bars are $0.5 \mathrm{~cm}$ for all photos. 

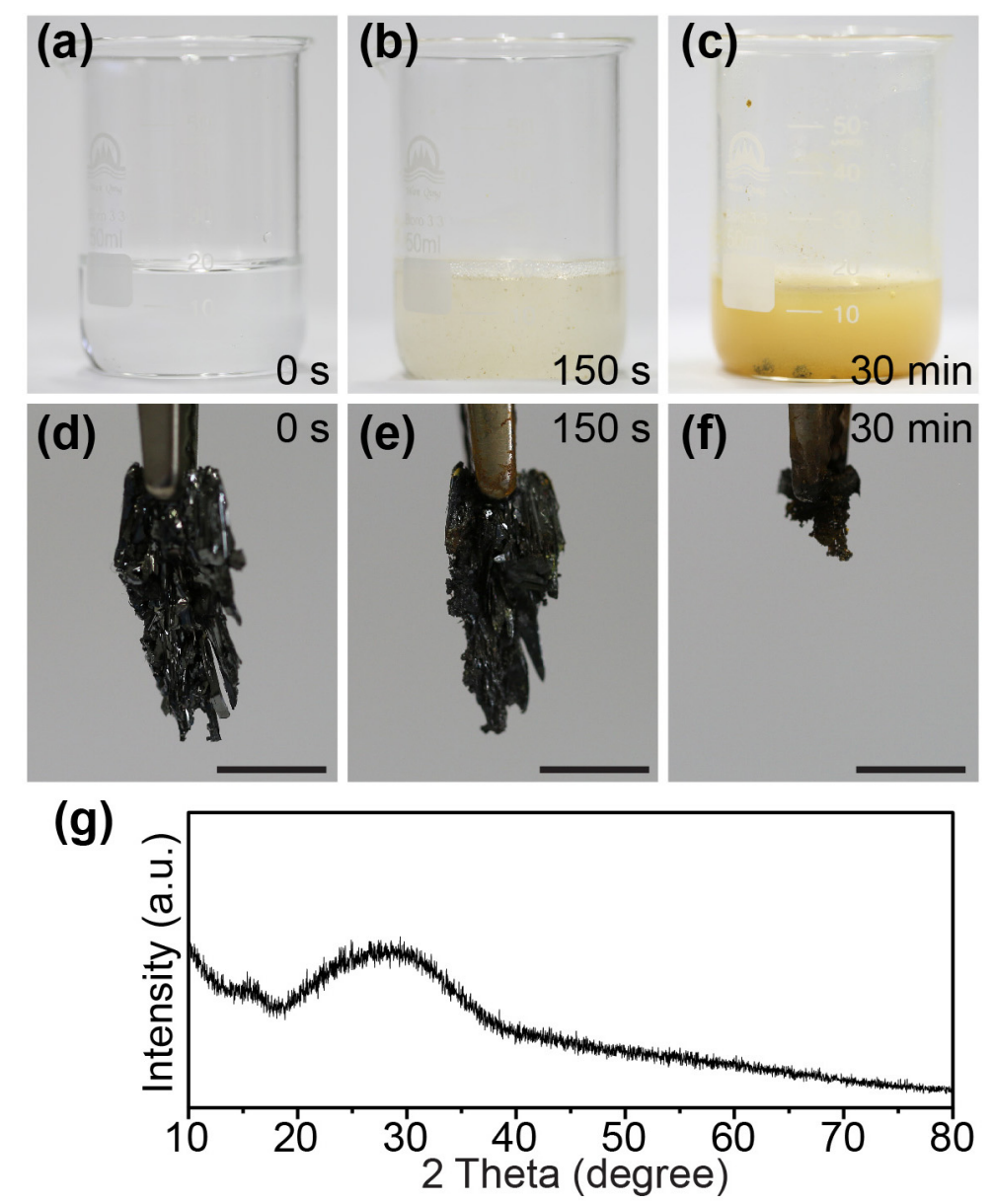

Figure S10. (a-c) Typical photos of the aqueous solution of $\mathrm{KCl}(0.5 \mathrm{M})$ after applying a voltage of $-30 \mathrm{~V}$ on the bulk BP cathode for different time at $50{ }^{\circ} \mathrm{C}$. (d-f) Typical photos of the bulk BP cathode after applying a voltage of $-30 \mathrm{~V}$ for different time in a $\mathrm{KCl}$ solution $(0.5 \mathrm{M})$ at $50^{\circ} \mathrm{C}$. Scale bars are $0.5 \mathrm{~cm}$ for $(\mathrm{d}-\mathrm{f})$. (g) Corresponding powder XRD pattern of the solid collected from the supernatant of (c).

After applying a voltage of $-30 \mathrm{~V}$ on the bulk BP cathode, in an aqueous solution of $\mathrm{KCl}(0.5 \mathrm{M})$ at $50{ }^{\circ} \mathrm{C}$, we observed continuous generation of electric sparks. Consequently, the solution gradually became yellow, and some parts of the bulk BP crystals were knocked off, setting down at the bottom of the beaker (Figure S10c). Meanwhile, no expansion of bulk BP crystals was observed (Figure S10d). After applying the voltage for 30 minutes, almost all bulk BP crystals were knocked off from the cathode (Figure S10f), which should be due to the electric sparks. Further analysis of the products in the aqueous solution, by powder XRD (Figure S10g), indicates that no bulk BP crystal was delaminated. Collectively, these results show that in the solution of $\mathrm{KCl}$, bulk BP crystals cannot be exfoliated, supporting our deduced functions of CTAC molecules. 

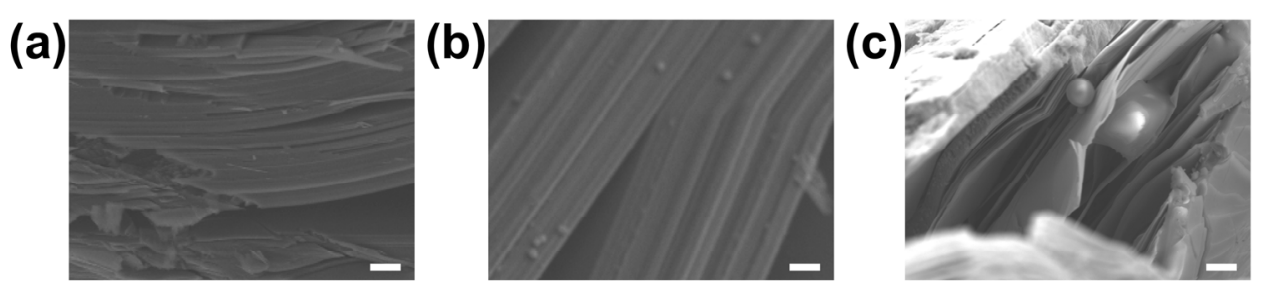

Figure S11. SEM images of the bulk BP crystals after applying a voltage of (a) -10 V, (b) $-20 \mathrm{~V}$, and (c) $-30 \mathrm{~V}$ for 10 minutes in an aqueous solution of $\mathrm{KCl}$ at $50{ }^{\circ} \mathrm{C}$, respectively. Scale bars are $1 \mu \mathrm{m}$ for all the images. 


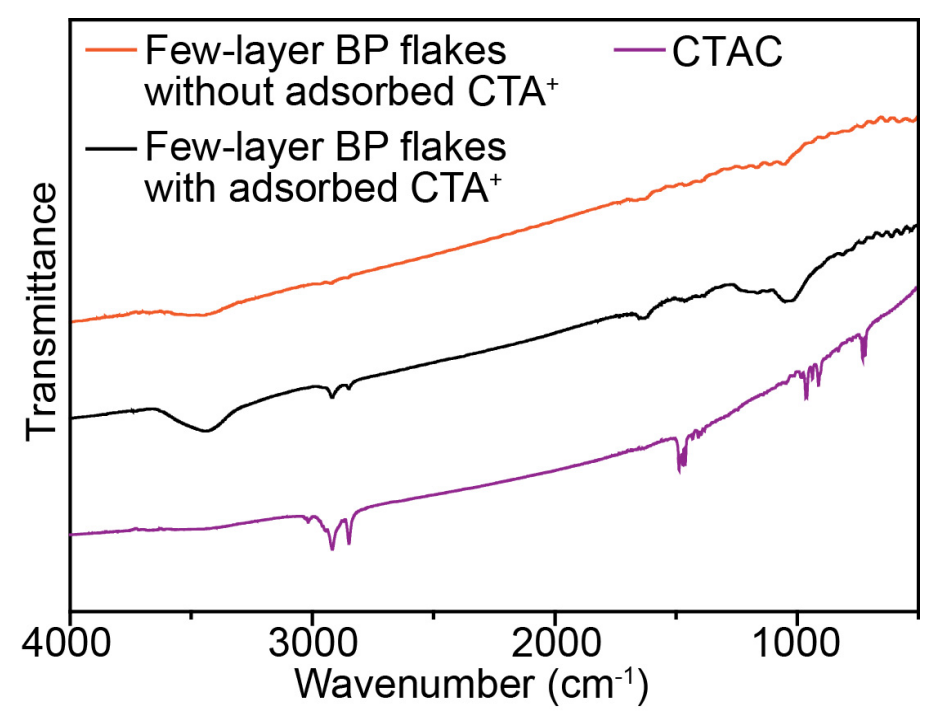

Figure S12. FTIR spectra of CTAC powder, few-layer BP flakes with adsorbed CTA ${ }^{+}$, and few-layer BP flakes without adsorbed $\mathrm{CTA}^{+}$, respectively. To remove the adsorbed $\mathrm{CTA}^{+}$, the as-exfoliated few-layer BP flakes were thoroughly washed with an ethanol solution of $\mathrm{NH}_{4} \mathrm{Cl}$ and isopropanol (details in Experimental Section: Post Treatment of Few-Layer Black Phosphorus). 


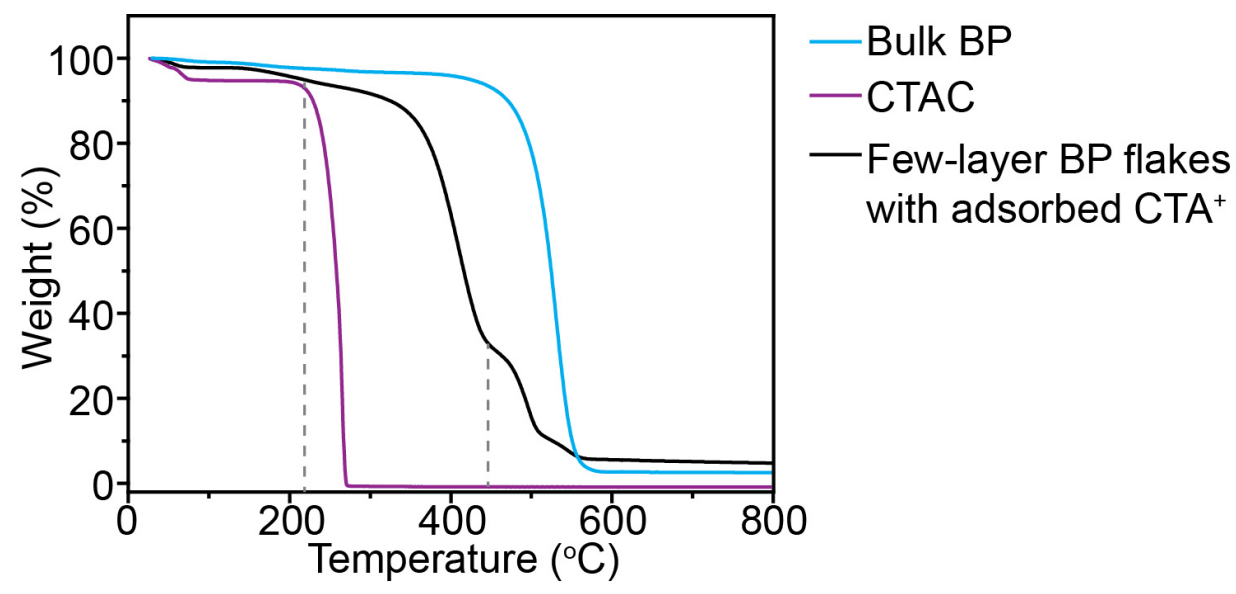

Figure S13. Thermogravimetric (TG) analysis of bulk BP, CTAC powder, and fewlayer BP flakes with adsorbed $\mathrm{CTA}^{+}$. According to the TG analysis, the weight loss of few-layer BP flakes with adsorbed $\mathrm{CTA}^{+}$, from $95 \%$ to $32 \%$ in the temperature range between 210 and $450{ }^{\circ} \mathrm{C}$ (indicated by the dashed lines in the figure), reveals the adsorption of $\mathrm{CTA}^{+}$on few-layer BP flakes. 

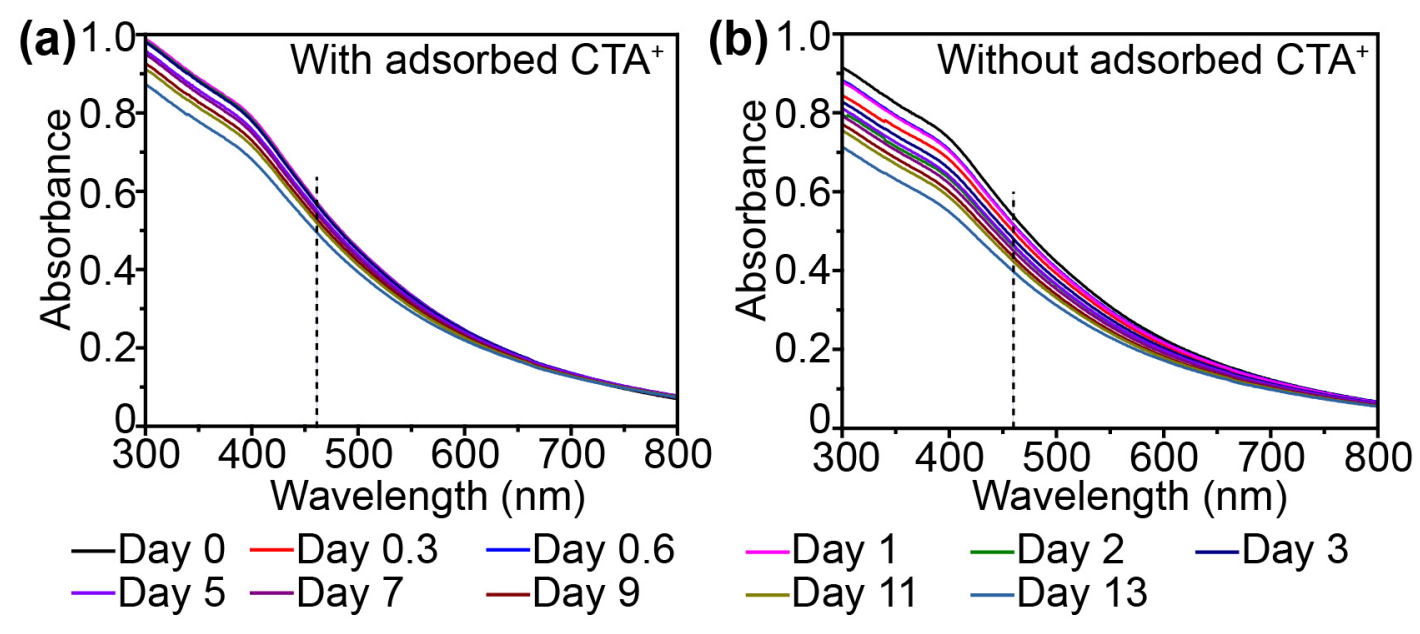

Figure S14. UV-vis absorption spectra of the dispersions of few-layer BP flakes stored for different time in the anoxic condition. Note that the dispersion used in (a) was prepared by dispersing the as-exfoliated few-layer BP flakes in isopropanol. For the dispersion used in (b), the as-exfoliated few-layer BP flakes were first washed thoroughly to remove the adsorbed $\mathrm{CTA}^{+}$(details in Experimental Section: Post Treatment of Few-Layer Black Phosphorus), and then dispersed in isopropanol for further measurements. The anoxic condition was created by purging $\mathrm{N}_{2}$ in the headspace of the vial prior to sealing by Parafilm. 

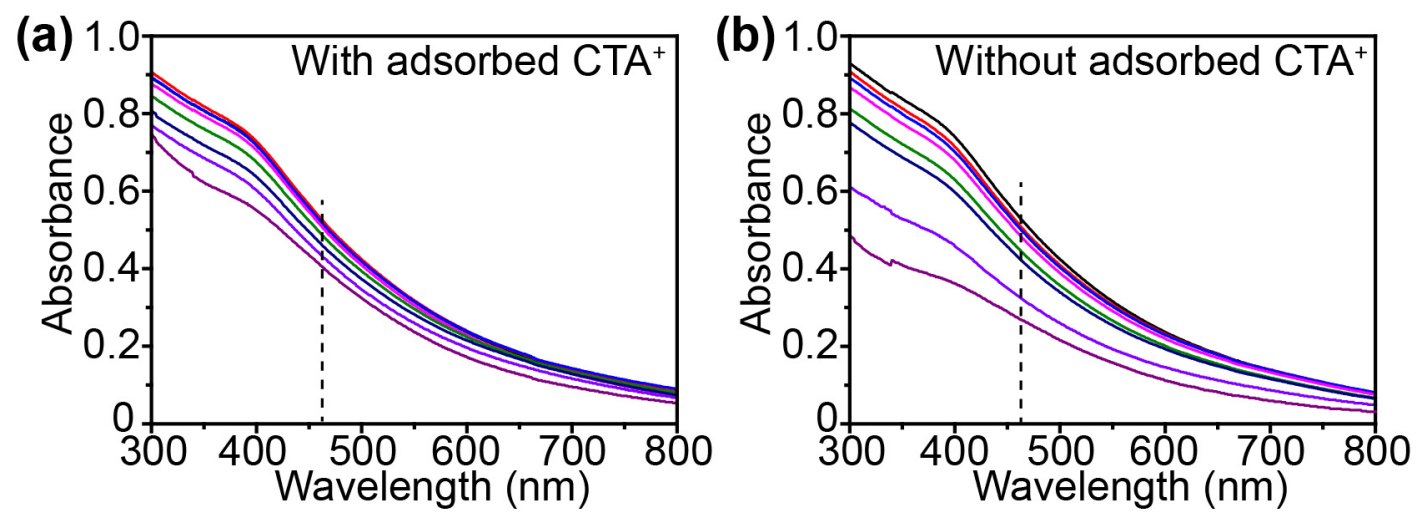

$$
\begin{array}{lll}
\text {-Day } 0 & \text {-Day } 0.3-\text { Day } 0.6-\text { Day } 1 \\
\text {-Day } 2 \text {-Day } 3 \text {-Day } 5-\text { Day } 7
\end{array}
$$

Figure S15. UV-vis absorption spectra of the dispersions of few-layer BP flakes stored for different time in the normal condition. Note that the dispersion used in (a) was prepared by dispersing the as-exfoliated few-layer BP flakes in isopropanol. For the dispersion used in (b), the as-exfoliated few-layer BP flakes were first washed thoroughly to remove the adsorbed $\mathrm{CTA}^{+}$(details in Experimental Section: Post Treatment of Few-Layer Black Phosphorus), and then dispersed in isopropanol for further measurements. The normal condition refers to the storage in a vial without $\mathrm{N}_{2}$ purging. 

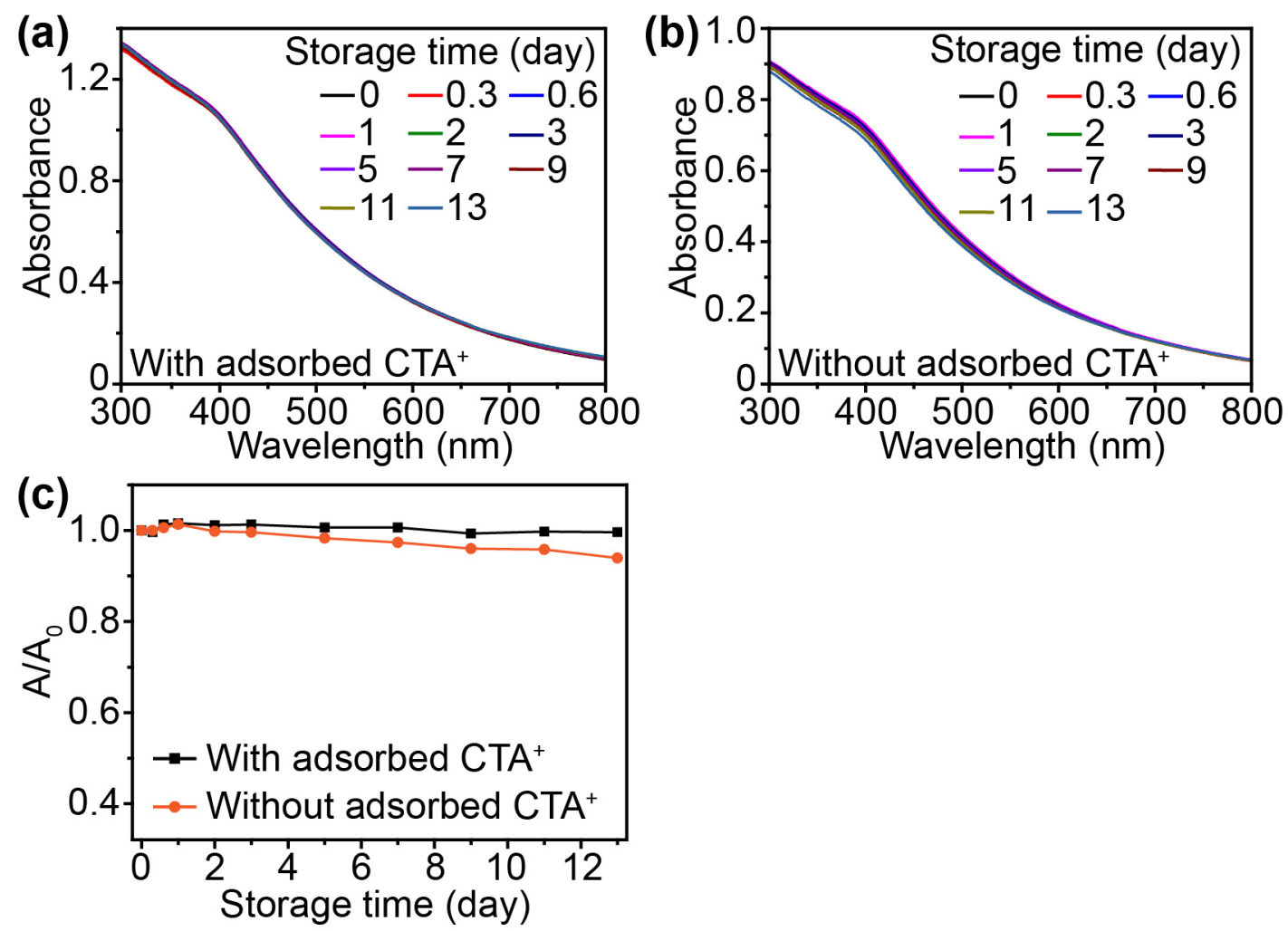

Figure S16. ( $a, b)$ UV-vis absorption spectra of the dispersions of few-layer BP flakes stored for different time in the anoxic and dark condition. Note that the dispersion used in (a) was prepared by dispersing the as-exfoliated few-layer BP flakes in isopropanol. For the dispersion used in (b), the as-exfoliated few-layer BP flakes were first washed thoroughly to remove the adsorbed $\mathrm{CTA}^{+}$(details in Experimental Section: Post Treatment of Few-Layer Black Phosphorus), and then dispersed in isopropanol for further measurements. The anoxic condition was created by purging $\mathrm{N}_{2}$ in the headspace of the vial prior to sealing by Parafilm. Subsequently, the dispersions were kept in a dark environment. (c) Storage time-dependent relative absorbance $\left(\mathrm{A} / \mathrm{A}_{0}\right)$ of few-layer BP dispersions under the anoxic and dark condition. A0 and A stand for the absorbance of the dispersions at $460 \mathrm{~nm}$ before and after storing for certain time, respectively. 


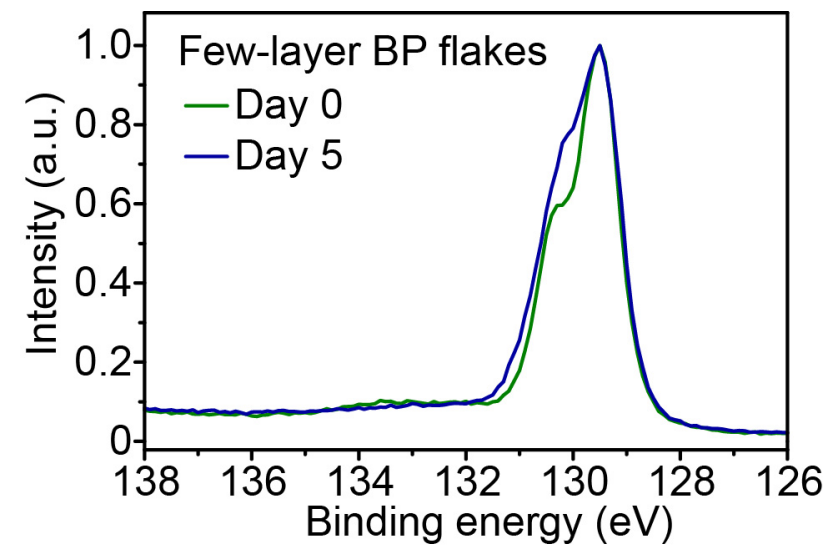

Figure S17. Normalized P 2p XPS spectra of the exfoliated few-layer BP flakes stored for different times in anoxic and dark condition. Note that the few-layer BP flakes with adsorbed $\mathrm{CTA}^{+}$were dispersed in isopropanol for storage. 
Table S1. Exfoliation of bulk BP under different conditions, corresponding phenomena, and optical band gaps of the products.

\begin{tabular}{|c|c|c|c|c|c|}
\hline $\operatorname{Exp}^{a}$ & $\begin{array}{c}\mathrm{T}^{b} \\
\left({ }^{\circ} \mathrm{C}\right)\end{array}$ & $\begin{array}{l}\text { Voltage } \\
\text { (V) }\end{array}$ & $\begin{array}{c}\mathrm{C}_{\mathrm{CTAC}^{c}} \\
(\mathrm{M})\end{array}$ & Phenomenon & $\begin{array}{l}\text { Optical } \\
\text { band gap }^{d} \\
(\mathrm{eV})\end{array}$ \\
\hline 1 & R.T. ${ }^{e}$ & -30 & 0.5 & Negligible exfoliation in $\sim 3 \mathrm{~h}$ & - \\
\hline 2 & 50 & -30 & 0.5 & Exfoliation started in $\sim 20 \mathrm{~s}$ & 2.454 \\
\hline 3 & 70 & -30 & 0.5 & Exfoliation started immediately & 2.464 \\
\hline 4 & 50 & -20 & 0.5 & No obvious change on bulk BP $f$ & $2.434^{g}$ \\
\hline 5 & 70 & -20 & 0.5 & Exfoliation started in $\sim 5$ min & 2.474 \\
\hline 6 & 50 & -10 & 0.5 & Negligible exfoliation in $\sim 30 \mathrm{~min}$ & - \\
\hline 7 & 70 & -10 & 0.5 & Negligible exfoliation in $\sim 30 \mathrm{~min}$ & - \\
\hline 8 & 50 & -30 & 0.1 & Exfoliation started in $\sim 15 \mathrm{~min}$ & - \\
\hline 9 & 70 & -30 & 0.1 & Exfoliation started in $\sim 10 \mathrm{~min}$ & - \\
\hline 10 & 50 & -30 & 0.7 & Exfoliation started in $\sim 27 \mathrm{~s}$ & - \\
\hline $\begin{array}{l}{ }^{a} \text { Exp.: } \\
\text { hexade } \\
\text { calcula } \\
\left.{ }^{\circ} \mathrm{C}\right) .{ }^{f} \mathrm{Tl} \\
\text { dark af }\end{array}$ & $\begin{array}{l}\exp \\
\text { trime } \\
\text { acco } \\
\text { TAC } \\
60 \mathrm{~m}\end{array}$ & $\begin{array}{l}\text { nent. } \\
\text { jlammor } \\
\text { ing to } \mathrm{U} \\
\text { blution } \mathrm{t} \\
\text { ites. }{ }^{\mathrm{g}} \mathrm{Th}\end{array}$ & $\begin{array}{l}\text { b } \mathrm{T} \text { : } \\
\text { Im chlo } \\
\text {-vis spe } \\
\text { ned slig } \\
\text { exfoliate }\end{array}$ & $\begin{array}{l}\text { emperature. }{ }^{c} \mathrm{C}_{\text {CTAC: }} \text { conce } \\
\text { ide (CTAC) solution. }{ }^{d} \text { The optical b } \\
\text { troscopy analysis. }{ }^{e} \text { R.T.: room ten } \\
\text { tly yellow after } 30 \text { minutes, and gra } \\
\text { d dispersion after reacting for } 60 \mathrm{mi}\end{array}$ & $\begin{array}{l}\text { ation of } \\
\text { d gaps were } \\
\text { erature }(\sim 22 \\
\text { lally became } \\
\text { tes was used }\end{array}$ \\
\hline
\end{tabular}

